\title{
Research on Cable Insulation Aging Under Acid-base Environment
}

\author{
Jin Ming Guo ${ }^{1}$ and Fei Feng Wang ${ }^{1}$ \\ ${ }^{1}$ Electric Power Research Institute of Guangxi Power Grid Co., Ltd, Nanning 530023, China
}

\begin{abstract}
In order to further study the effect of different degree of acidity and alkalinity on cable insulation aging, the aging condition of cable insulation was compared and analyzed under different $\mathrm{pH}$ values. The samples of cable insulation material slice and short cable were carried out the accelerated aging test, which were placed in the conditions of different $\mathrm{pH}$ values. And then the test results of micro morphology and electrical properties between the different samples were compared and analyzed after aging. According to the test results in this paper, the micro morphology of the cable insulation material slice would change differently under the aging conditions of different acidity and alkalinity, which indicated that the degree of oxidation degradation of insulation material aging was different from the different acidity and alkalinity conditions. In addition, both acid or alkaline environment would accelerate the aging of cable insulation and reduce the service life of cable, while strong acid and strong alkaline environment are more conducive to the formation of interpenetrating water tree in cable insulation material and cause the operation accident of power cable. Furthermore, the test results of electrical properties of short cable samples after aging showed that the aging degree of short cable samples was more serious in alkaline environment, and the decline of electrical properties was faster.
\end{abstract}

\section{Introduction}

Because of the good mechanical and electrical properties of cross-linked polyethylene (XLPE), the XLPE power cables are widely used in medium and low voltage transmission lines in China. With the improvement of the utilization rate of cables and the continuous growth of the service life, cable accidents caused by insulation aging are becoming more and more serious[1-3]. China has a vast land area, and different laying environments have different effects on cable aging [4-5]. Therefore, it is of great significance to study the influence of cable operation environment on cable insulation aging.

Nowadays, a large number of documents have clarified that contact with electrolytic ions contained in the dielectric is one of the important influencing factors of cable insulation aging when the cable is running[6-8]. The dielectric in acidic or alkaline environment mostly contains electrolytic ions. And the higher the concentration of electrolytic ions, the easier it is to enter into the cable insulation, which would impact the molecular chain or lead to the oxidative decomposition of the molecular groups in the insulation material, thus accelerating the aging of the cable insulation[8-10]. With the increase of cable service life, the underground cables will inevitably be affected by water immersion and acidbase substances corrosion, resulting in damage to external protection and accelerated aging of internal insulation. The $\mathrm{pH}$ value of the dielectric contacted by cable is directly related to the degree of cable corrosion and its electrical performance[11]. Due to the differences of climate and soil characteristics, the accelerated aging degree of cables in different regions is different. Therefore, it is of great significance to study the influence of acid-base environment on the insulation performance of cables. At present, there is no research report on the influence of acid-base environment on cable insulation.

In this paper, the accelerated aging experiments of cable insulation material and power cable sample were carried out under different acid-base condition. Based on the experimental results, the aging degree of insulating material under different acid-base conditions was analyzed. And the influence of different acid-base environment on cable operation was discussed.

\section{Sample and experiment}

\subsection{Aging test of XLPE slice}

In order to study the influence of environmental $\mathrm{pH}$ value on cable insulation aging, firstly, the water tree aging characteristic of XLPE in acid-base environment was studied. The XLPE slice crosslinked by peroxide and stifled under the processing condition of industrial $10-\mathrm{kV}$ cable insulation material was selected as the experimental sample, therefore, its dielectric properties were similar to the insulation material of industrial XLPE cable. The XLPE slice was made into a 
rectangular sample of $50 \mathrm{~mm} \times 50 \mathrm{~mm} \times 3 \mathrm{~mm}$, which the area with a diameter of $25 \mathrm{~mm}$ in the middle of the sample was taken as the accelerated water tree aging area. The 18 pinhole defects with a depth of $1.5 \mathrm{~mm}$ in three rows were evenly pierced into the aging area with steel needle.

The accelerated water tree aging test was carried out with cup-shaped experimental device recommended by IEC/TS 61956 standard, as shown in Figure 1. The XLPE sample was fixed between the cup body and the copper electrode at the bottom of the cup. The aging solution was added into the cup, and then the cup cover was covered. The $\mathrm{pH}$ values of the aging solution were respectively determined as $1,5,9$ and 13.Then, a sinusoidal AC voltage with effective value of $7.5 \mathrm{kV}$ and frequency of $400 \mathrm{~Hz}$ was applied to the upper copper electrode, when the copper electrode at the bottom of the cup was grounded.

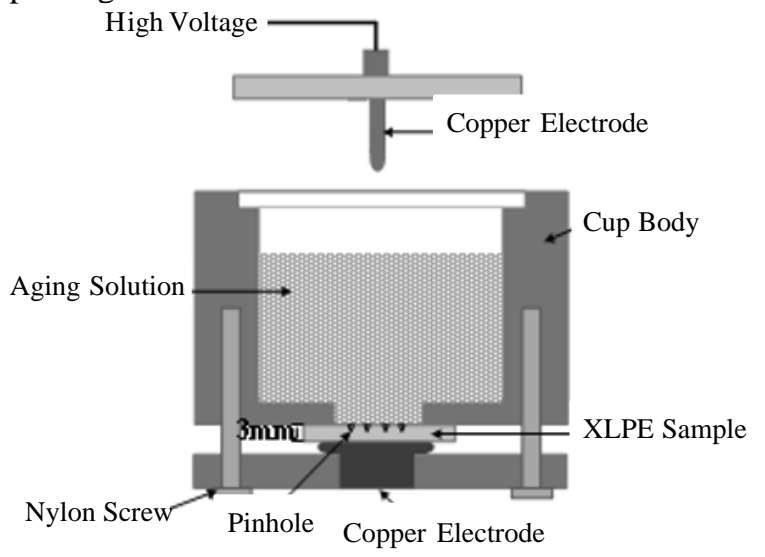

Fig.1 Diagram of test cell assembly

\subsection{Aging test of short cable}

In order to study the influence of environmental $\mathrm{pH}$ value on cable insulation performance, the YJLV22$3 \times 958.7 / 15 \mathrm{kV}$ XLPE cable was selected to carry out artificial accelerated aging test. The schematic diagram of accelerated aging device platform for short cable is shown in Figure 2. The two ends of the outer heat shrinkable tube of the short cable sample were sealed, and the solution with different $\mathrm{pH}$ values were added into the tube to ensure the immersion of pinhole defects during the accelerated aging process. After aging for 4 weeks, the dielectric loss and polarization and depolarization current (PDC) results of short cable samples were measured. Multiple cable samples were aged in each group of solution at the same time, and the average value of the measured results was taken for comparison and analysis.

\section{Results and discussion}

\subsection{Aging analysis of XLPE slice}

After aging, the XLPE samples were cut into $100 \mu \mathrm{m}$ thick slices with a slicer. The slices were dyed in methylene blue solution at $90^{\circ} \mathrm{C}$ for $30 \mathrm{~min}$, and then the morphology of water tree was observed with an optical microscope and the initiation rate of water tree was counted.

The initiation rate of water tree is calculated by equation (1):

$$
I=\frac{n_{1}}{n} \times 100 \%
$$

Where, $n_{1}$ is the number of pinhole initiating water tree; $n$ is the total number of pinhole.

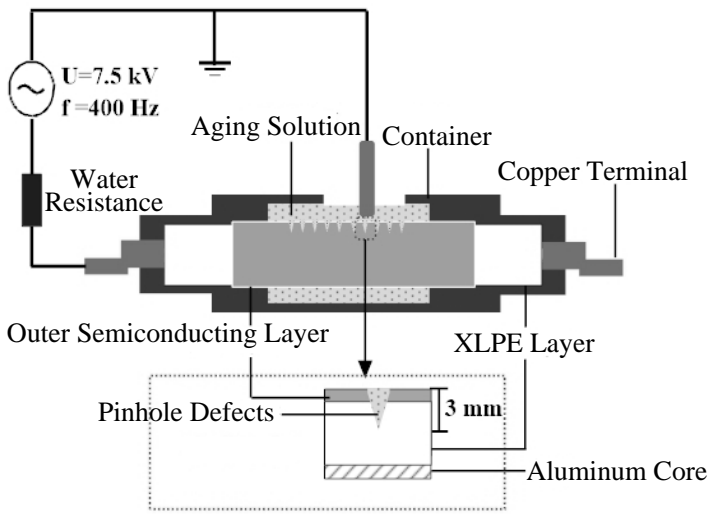

Fig.2 Schematic diagram of cable accelerated aging

According to the observation results, the initiation rate of water tree in each aging solution was $100 \%$. The micro morphology of water tree was shown in Fig.3, and the aging morphology of water tree under different $\mathrm{pH}$ values was quite different. The staining of water tree area was deeper in acid environment, but lighter in alkaline environment. The staining degree of insulating materials depends on the number of micro-hole generated by material degradation in the aging area. The number of micro-hole is more, methylene blue solution is easier to adhere, otherwise it is not easy to be stained. Therefore, the staining of water tree area indicates that the degree of aging degradation of insulating materials around the initial point of aging is more serious under acidic condition.

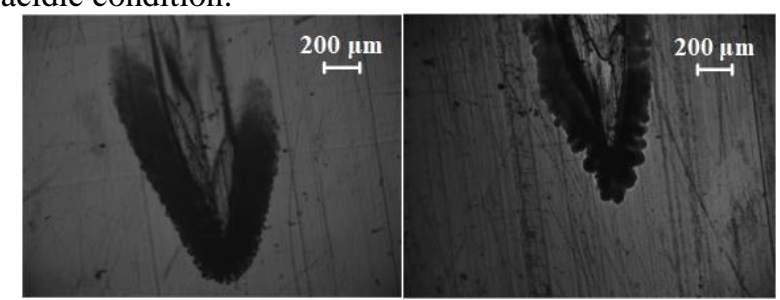

(a) $\mathrm{pH}=1$

(b) $\mathrm{pH}=5$

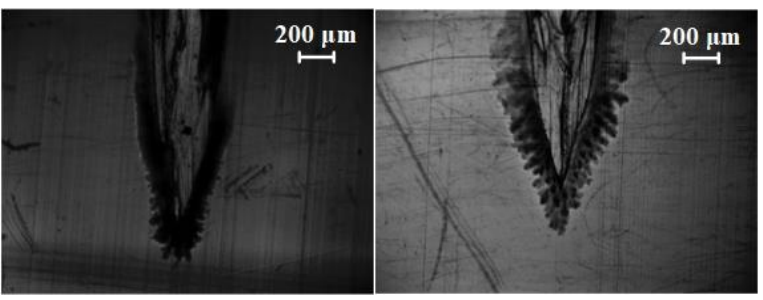

(b) $\mathrm{pH}=9$

(d) $\mathrm{pH}=13$

Fig.3 Morphology of the water tree in XLPE slice

In order to further analyze the aging changes of insulating materials under different acid-base conditions, the water tree dimensions at the front end of the needle 
tip and both sides of the pinhole were measured. As shown in Figure 4, when $\mathrm{pH}=1$, the length of water tree at the needle tip is $199 \sim 205 \mu \mathrm{m}$, and the average length is $202.04 \mu \mathrm{m}$. When $\mathrm{pH}=5$, the length of water tree at the needle tip is $200 \sim 270 \mu \mathrm{m}$, and the average length is $236.83 \mu \mathrm{m}$. When $\mathrm{pH}=9$, the length of water tree at the needle tip is $210 \sim 260 \mu \mathrm{m}$, and the average length is $235.92 \mu \mathrm{m}$. When $\mathrm{pH}=13$, the length of water tree at the needle tip is $250 \sim 300 \mu \mathrm{m}$, and the average length is $267.55 \mu \mathrm{m}$.

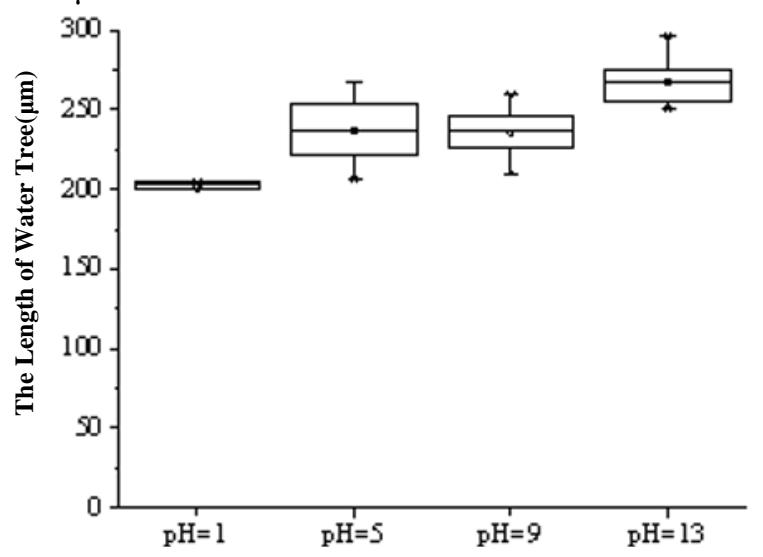

Fig.4 Length of water tree at the needle tip

The size of the water tree on both sides of the pinhole is shown in Figure 5. When $\mathrm{pH}=1$, the size of water tree is $200 \sim 230 \mu \mathrm{m}$, and the average width is $218.02 \mu \mathrm{m}$. When $\mathrm{pH}=5$, the size of water tree is $170 \sim 200 \mu \mathrm{m}$, and the average width is $191.24 \mu \mathrm{m}$. When $\mathrm{pH}=9$, the size of water tree is $100 \sim 130 \mu \mathrm{m}$, and the average width is $118.50 \mu \mathrm{m}$. When $\mathrm{pH}=13$, the size of water tree is $170 \sim$ $230 \mu \mathrm{m}$, and the average width is $205.34 \mu \mathrm{m}$.

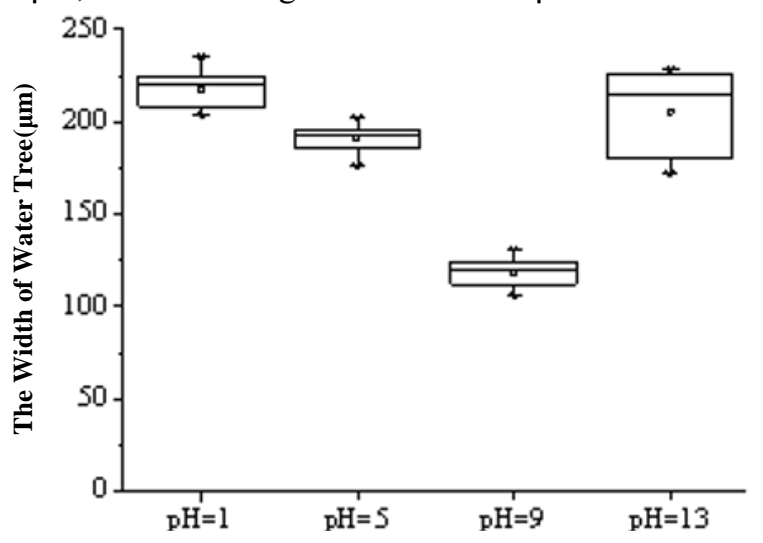

Fig.5 Width of water tree on both sides of pinhole

Analyzing the size of water tree on both sides of pinhole discovered that the width of water tree was almost proportional to the acid-base degree of solution. Compared with weak acid and weak base, the width of water tree under strong acid and strong base is larger, and the difference of water tree width under $\mathrm{pH}=1$ and $\mathrm{pH}=13$ is small. Comparing the size of water tree under alkaline condition, it was found that the size of water tree at the needle tip was significantly higher than that on both sides. Therefore, the acid-base degree of aging environment is an important factor which will affect the length of water tree. The formation of penetrating water tree or water tree developing into electricity tree will lead to insulation failure, and then cause cable operation accident[12-13]. The experimental results show that the water tree is easier to grow along the direction of electric field line under strong acid-base condition, thus forming penetrating water tree which has serious harm to the operation of the cable.

According to the electric-mechanical theory of water tree growth, the defects such as impurities, cracks, bubbles and semi conductive layer bulge in polymer materials will form local high field strength under the action of voltage. Water molecules, ions and so on will move to the concentrated part of the electric field to form dielectrophoresis. Under the action of periodic Maxwell stress, ions can cause the molecular chain to break and generate small cracks which will continuously develop to form water tree[14]. The formula of Maxwell stress is:

$$
F=\left(\varepsilon_{0} / 2\right) \nabla\left(\varepsilon_{\mathrm{r}}-1\right) E^{2}
$$

Where: $\varepsilon_{0}$ is the vacuum dielectric constant; $\varepsilon_{\mathrm{r}}$ is the relative dielectric constant; $E$ is the electric field strength[15].

There are a lot of ions in water under acid-base condition. Under the action of Maxwell stress, ions and water molecules diffuse along the needle tip defects and enter the amorphous region. Due to the continuous action of AC electric field, ions and water molecules will exert periodic Maxwell stress on the surrounding XLPE material. When the exerted energy exceeds the bond energy of XLPE material molecular chain, the molecular chain will break and form local micro-cracks. The crack develops continuously and then forms water branches which will lead to the aging of insulation layer. In addition, the ion concentration of $\mathrm{pH}=1$ and $\mathrm{pH}=13$ in the aging solution is higher than that of $\mathrm{pH}=5$ and $\mathrm{pH}=9$. Because a large number of ions have strong destructive effect on the molecular chain of cross-linked polyethylene under Maxwell stress, the size of water tree formed is large. Therefore, the size of water tree under strong acid-base condition is larger than that under weak acid-base condition.

\subsection{Experiment result and analysis of short cable}

In order to further simulate the influence of acid-base environment on the electrical performance of cable in actual operation, the short cable samples were accelerated aged at $\mathrm{pH}=1,5,9$ and 13. After 4 weeks, PDC low frequency loss and power frequency dielectric loss test methods were used to test the electrical performance of short cable samples.

According to IEEE Std 400tm-2001, when the dielectric loss factor is less than $1.2 \times 10^{-3}$ at $0.1 \mathrm{~Hz}$, the cable insulation is good. As shown in Table 1 , when $\mathrm{pH}=1,5,9$, the $0.1 \mathrm{~Hz} \tan \delta(\%)$ value of short cable sample was between $1.8-2.1$, which indicated that the cable insulation existed aging phenomenon. When $\mathrm{pH}=13$, the $0.1 \mathrm{~Hz} \tan \delta(\%)$ value of short cable sample was 2.7339 , which indicated that the deterioration of 
cable insulation was more serious, and it was urgent to replace or take further disposal measures.

Table 1. Test results of electrical performance of short cable samples after aging.

\begin{tabular}{|c|c|c|}
\hline $\mathrm{pH}$ value & $0.1 \mathrm{~Hz} \tan \delta(\%)$ & $50 \mathrm{~Hz} \tan \delta(\%)$ \\
\hline 1 & 1.8295 & 0.622 \\
\hline 5 & 1.8883 & 0.141 \\
\hline 9 & 2.0968 & 0.149 \\
\hline 13 & 2.7339 & 0.823 \\
\hline
\end{tabular}

On the other hand, when the water tree aging occurs in the insulation layer of power cable, the insulation performance of the cable will gradually decrease with the growth of water tree defects, and then the corresponding $\tan \delta$ value will gradually increase. As shown in Table 1 , the $\tan \delta$ value of short cable samples under strong acid-base condition was significantly higher than the value under weak acid-base condition. And the $\tan \delta$ value of short cable samples at $\mathrm{pH}=13$ was also higher than the value at $\mathrm{pH}=1$. The results of PDC lowfrequency loss test and power frequency dielectric loss test of short cable samples under different degree of acid-base conditions were analyzed comprehensively. It was found that the electrical performance of cable samples aged under alkaline condition decreased more obviously.

\section{Conclusion}

In this paper, the accelerated aging experiment of insulation material slice and short power cable samples were carried out under different degree of acid-base conditions. By observing and analyzing the changes of water tree morphology, water tree length and electrical performance, the following conclusions are obtained:

a) The water tree size under strong acid-base condition is larger than that under weak acid-base condition. The length of needle tip water tree under alkaline condition is longer than that under acidic condition. And the length of water tree at the needle tip is longer than that on both sides of pinhole. Therefore, the water tree in cable insulation is easier to extend along the direction of electric field line under alkaline condition, which will form the penetrating water tree and have more serious harm to the actual operation of the cable.

b) Comparing the results of PDC low-frequency loss test and power frequency dielectric loss test of short cable samples aged under different acid-base conditions, it is found that the electrical performance of the short cable samples decreases more obviously after aging under alkaline condition. Combined with the experimental results of the slice samples, it further shows that the influence of alkaline environment on the insulation aging of power cable is more obvious.

\section{References}

1. Chen Tao, et al. Mechanism, Detection and Prevention of Water Treeing in XLPE Power Cables[J]. Electric Wire \& Cable, 2009(4): 1-7.

2. Boggs S, Xu J. Water Treeing-filled Versus Unfilled Cable Insulation[J]. IEEE Electrical Insulation Magazine, 2001, 17(1): 23-29.

3. Wang Z, Marcolongo $\mathrm{P}$, Lemberg $\mathrm{J}$ A, et al. Mechanical Fatigue as a Mechanism of Water Tree Propagation in TR-XLPE[J]. IEEE Transactions on Dielectrics and Electrical Insulation, 2012, 19(1): 321-330.

4. Mao Wen-pei, et al. Investigation of Installation and Type Selection of Cables at Low Temperatures[J]. Electric Wire \& Cable, 2009(5): 38-40.

5. Liu Zhi-yong. Discussion on the maintenance method of cable line [J]. CHENG SHI ZHAO MING, 2011(4): 54-55.

6. He Jun, TU De-min. Growth Mechanism and Inhibition Methods of Water Treeing in XLPE Cable Insulation[J]. Insulating Materials, 2008, 41(6): 54-58.

7. Wu Song-zhen. Effect of multi-factor synergy on environmental aging of insulating materials[J]. Electrical application, 1986(3) :3-7.

8. Zhao Jian-kang, et al. Review of Influence of Water-tree on Microstructure and Properties of XLPE Cable Insulation Material[J]. Insulating Materials, 2010, 43(5):50-56.

9. Hou Zhen-qi, et al. Study on Water Tree Propagation in XLPE Power Cables by Molecular Chains Fatigue Fracture Theory[J]. Insulating Materials, 2016(10):53-59.

10. Zhou Kai, et al. Toward Understanding the Characteristics of Water Tree Growth at Different Temperatures in XLPE Based on the Theory of Molecular Orientation[J]. High Voltage Engineering, 2014, 40(12): 3665-3673.

11. Zhang Zong-xi, et al. Research and Application of Power Cable Insulation Overall Aging Test Technology[J]. China Electric Power (Technology Edition), 2016, 2: 49-51.

12. Zhen Xiao-quan, et al. Transformation of Electrical Tree from Water Tree Degradation in XLPE[J]. Proceedings of the CSEE, 2013, 36(22): 166-174.

13. Yuan Jian, et al. Study on Mechanism of Water Tree Caused in XLPE Power Cable Dielectric[J]. High Voltage Engineering, 2002, 28(2): 8-9.

14. Abderrazzaq M H. Development of Water Tree Structure in Polyester Resin[J]. IEEE Transactions on Dielectrics and Electrical Insulation, 2005, 12(1): 158-165.

15. Wang Z, Marcolongo P, et al. Mechanical Fatigue as a Mechanism of Water Tree Propagation in TRXLPE[J]. IEEE Transactions on Dielectrics and Electrical Insulation, 2012, 19(1): 321-330. 